\section{Case Report}

Journal of Epilepsy Research pISSN 2233-6249 / elSSN 2233-6257

Received June 8, 2021

Revised November 4, 2021

Accepted November 4, 2021

Corresponding author:

Jung-Won Shin, MD, PhD

Department of Neurology, CHA Bundang

Medical Center, CHA University, 59

Yatap-ro, Bundang-gu, Seongnam 13496,

Korea

Tel. $+82-31-780-5058$

Fax. +82-31-780-3449

E-mail; limitsum@gmail.com

\title{
Early Combination Therapy of Ketamine and Midazolam in Patients with Refractory Status Epilepticus in Hemodynamic Unstable State
}

\author{
Jung-Won Choi, MD, Jung-Won Shin, MD, PhD \\ Department of Neurology, CHA Bundang Medical Center, CHA University, Seongnam, Korea
}

\begin{abstract}
The use of anesthetics is inevitable to suppress seizure activity in refractory status epilepticus (RSE). Hypotension, which is a critical side effect observed when treating RSE using a higher dosage of anesthetics that enhance $y$-aminobutyric acid (GABA) activity, often requires vasopressor agents. Concomitant treatment with N-methyl-D-aspartate (NMDA) receptor antagonists, such as ketamine, could be effective in prolonged refractory SE, while maintaining stable blood pressure owing to the blockage of catecholamine reuptake in the systemic circulation. We report two cases of patients who had RSE with hemodynamic instability treated promptly with an early combination of ketamine and low-dose midazolam. The combination treatment effectively suppressed epileptic discharge with less hemodynamic side effects; moreover, a low dose of midazolam was required when combined with ketamine therapy. The initial combination of a third-line therapy that blocks NMDA receptors with enhanced GABAergic activity could be useful in RSE. Further studies are necessary in many variable etiologies of SE. (2021;11:150-153)
\end{abstract}

Key words: Ketamine, Status epilepticus, Combined modality therapy

\section{Introduction}

Refractory status epilepticus (RSE) is defined as persistent seizures that are resistant to first-line and second-line antiepileptic drugs (AEDs), usually requiring general anesthesia and continuous electroencephalography (EEG) monitoring. ${ }^{1}$ Current guidelines recommend prompt organized treatment first using benzodiazepines, followed by a sequential addition of second-line drugs if status epilepticus (SE) persists. ${ }^{2}$ However, in experimental models, during prolonged seizures, $\gamma$-aminobutyric acid agonist (GABA $A_{A}$ ) receptors are internalized, while glutamate receptors (mainly N-methyl-D-aspartate [NMDA] receptors) increase their concentration at the synapse. Hence, benzodiazepine resistance could also be attenuated by other potential mechanisms, including changes in other ion channels such as sodium or potassium and cholinergic mechanisms. ${ }^{3}$ Therefore, the pathophysiology of SE suggests that the most effective pharmacologic intervention is likely to be early polytherapy, which possesses different mechanisms of action.

In several animal and human studies, early polytherapy composed of a benzodiazepine and a second-line drug, including ketamine or levetiracetam, which modulates glutamate receptors, seems to be a practical approach. ${ }^{4}$ Certain studies on early anesthetic combination therapy for RSE or super RSE, such as midazolam-pentobarbital or propofol-ketamine, have shown favorable outcomes. ${ }^{5,6}$ An early use of ketamine for the treatment of $\mathrm{SE}$, mainly in combination with other drugs, is being studied in animal models with promising results; studies showed that ketamine is remarkably neuroprotective through NMDA receptor blockade even when given following the onset of SE. ${ }^{7}$ Moreover, ketamine shows fewer cardiovascular side effects than other anesthetics. ${ }^{1}$ We report two cases of RSE with hemodynamic instability due to alcohol withdrawal syndrome and septic shock treated with early combination therapy of ketamine and low-dose midazolam, with favorable outcomes.

\section{Case Report}

\section{Case 1}

A 46 years old female was admitted to the emergency department 
in comatose state accompanied by sustained convulsive generalized tonic-clonic seizures. She had a history of alcoholic liver cirrhosis and depression with heavy chronic alcoholism and took AEDs, including levetiracetam and clobazam twice daily and lorazepam once daily owing to alcohol-related seizures a year ago. In the emergency room, intravenous lorazepam $4 \mathrm{mg}$ was administered immediately. Although the tonic-clonic seizures terminated, focal seizures with jerking movements of the left arm and legs were not controlled, and there was no recovery in consciousness. Intra-cerebral hemorrhage on the initial brain computed tomography (CT) scan was absent. The body temperature was $39.8^{\circ} \mathrm{C}$. Initial laboratory findings showed mild leukocytosis $(16,200 / \mu \mathrm{L})$ with elevated C-reactive protein (CRP, $2.59 \mathrm{mg} / \mathrm{dL})$, and elevation of the aspartate aminotransferase (AST) and alanine aminotransferase (ALT) levels. Serum glucose and electrolytes with ionized calcium were within the normal range. Arterial blood gas analysis revealed severe acidosis with carbon dioxide retention. An endotracheal tube was inserted and mechanical ventilation was performed because of compromised respiratory dysfunction with an abrupt decrease in oxygen saturation. Thereafter, intravenous fosphenytoin (20 mg/kg) and levetiracetam (3,000 mg) were administered. Brain magnetic resonance imaging (MRI) revealed high signal intensities in the right hemisphere in T2 fluid attenuated inversion recovery (FLAIR), which suggested seizure-related changes. After shifting to the intensive care unit, continuous video EEG monitoring was performed, which showed a
A

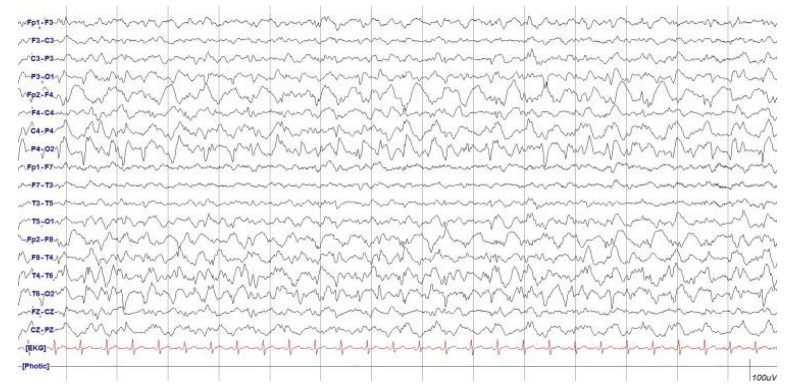

B

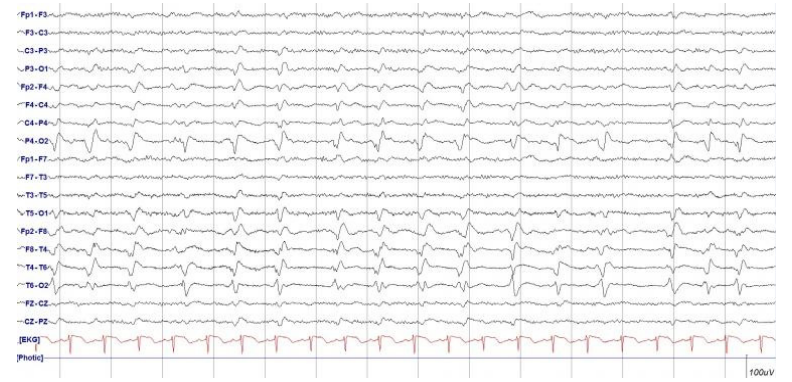

Figure 1. The continuous electroencephalography recording shows (A) nearly continuous 1-Hz rhythmic sharp waves in the right hemisphere (patient 1) and (B) 1-Hz periodic lateralized epileptiform discharges in the right hemisphere (patient 2).

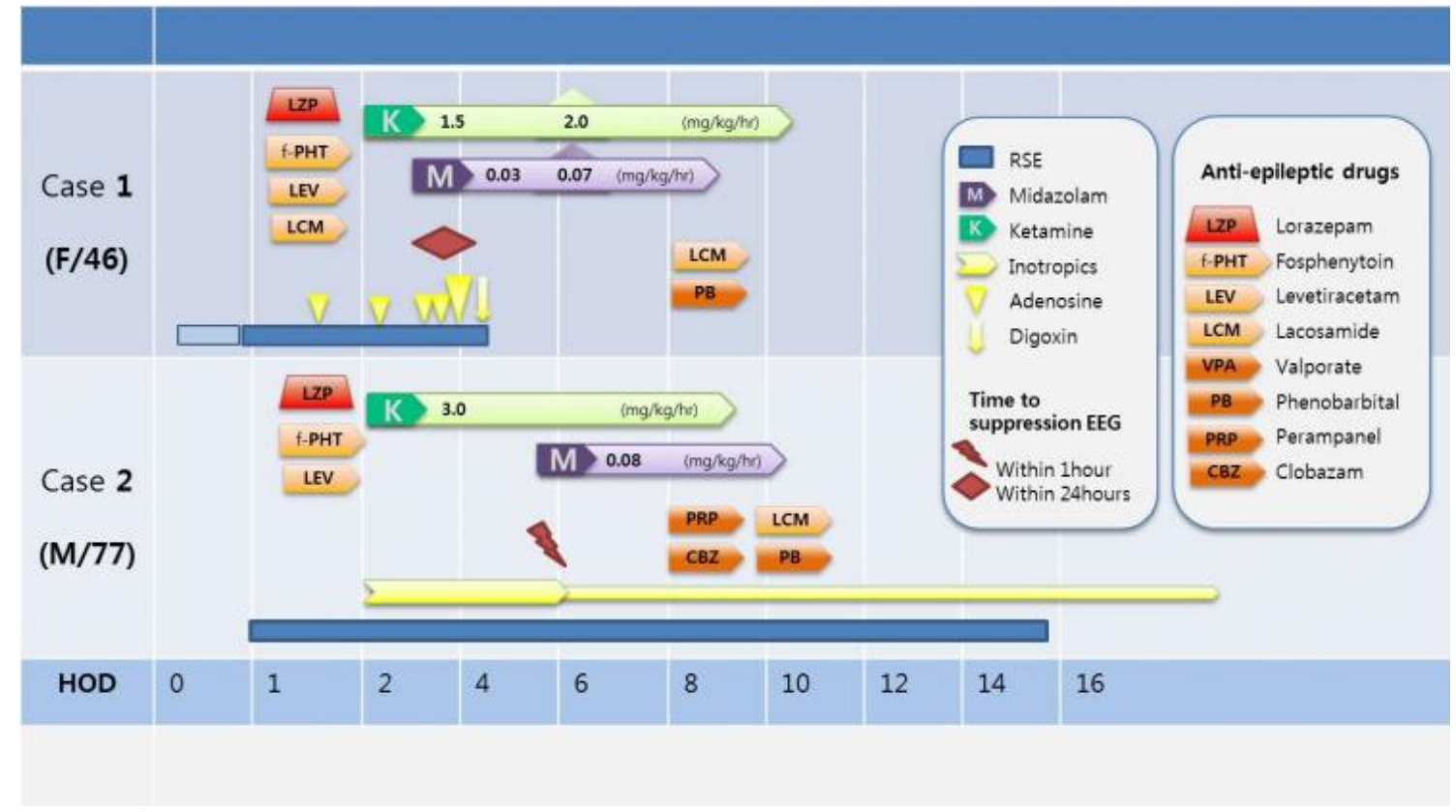

Figure 2. Detailed treatment and clinical course of two patients with refractory status epilepticus treated with ketamine-midazolam combination therapy. First line to third line antiepileptic drugs administered during the treatment course are presented schematically. HOD, hospital day; RSE, refractory status epilepticus; EEG, electroencephalogram. 
nearly continuous 1-Hz rhythmic sharp waves in the right temporo-occipital areas (Fig. 1A). The patient did not respond to first-and second-line treatment regimens for SE and presented with autonomic dysfunctions, including tachycardia (160-230 beats/min) and fluctuating blood pressure due to alcohol withdrawal syndrome. Third line treatment for SE as low-dose combination therapy with ketamine and midazolam was initiated. Continuous intravenous ketamine $1.5 \mathrm{mg} / \mathrm{kg} / \mathrm{h}$ up to $2 \mathrm{mg} / \mathrm{kg} / \mathrm{h}$ infusion with intravenous midazolam $0.03 \mathrm{mg} / \mathrm{kg} / \mathrm{h}$ up to $0.07 \mathrm{mg} / \mathrm{kg} / \mathrm{h}$ were administered for the management of unstable hemodynamic status (Fig. 2).

Rhythmic sharp and waves on EEG disappeared within 24 hours of combination continuous midazolam and ketamine infusion, and autonomic dysfunctions were controlled. After continuous combination infusion for 48 hours, both regimens were tapered out with concomitant intravenous phenobarbital $20 \mathrm{mg} / \mathrm{kg}$ loading and a maintenance dose of $30 \mathrm{mg}$ three times a day. Upon improvement of the EEG findings with recovered consciousness, the patient was treated with levetiracetam $750 \mathrm{mg}$, phenytoin $150 \mathrm{mg}$, lacosamide $150 \mathrm{mg}$, and phenobarbital $30 \mathrm{mg}$ twice daily. The patient was successfully weaned off the ventilator on hospital day 8 and continued with physical therapy to return to her baseline (modified Rankin score improved from 5 to 1).

\section{Case 2}

A 77-year-old man was admitted to the emergency room with acute stuporous mental change preceding generalized tonic-clonic seizures of at least 20 minutes. He had a history of angina and atrial fibrillation. He was a chronic alcoholic and had no history of seizures or other major neurological illnesses. He was promptly administered intravenous lorazepam (total $8 \mathrm{mg}$ ), followed by 3,000 mg levetiracetam and $20 \mathrm{mg} / \mathrm{kg}$ fosphenytoin sodium. Convulsive seizures ceased; however, the mental status was still stupor. The body temperature was $39^{\circ} \mathrm{C}$. Initial laboratory tests showed leukocytosis $(10,610 / \mu \mathrm{L})$ with elevated CRP (3.32 mg/dL) and mildly elevated AST and ALT levels. Other laboratory tests, including glucose, electrolytes, and ammonia, were within the normal range. Arterial blood gas analysis showed mild metabolic acidosis. Cerebrospinal fluid analysis showed no white blood cells (WBCs), with normal glucose and protein concentrations. Blood gram staining and bacterial culture showed positive results for Staphylococcus aureus. No intra-cerebral hemorrhage was observed on the initial brain CT scan: however, liver cirrhosis and basal pneumonia were reported. Pancytopenia and markedly increasing CRP levels

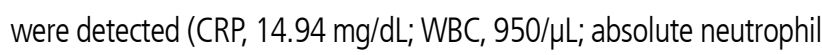

count, 853.6/ $\mathrm{LL}$; hemoglobin, $11.6 \mathrm{~g} / \mathrm{dL}$; and platelet, 103,000/ $\mathrm{LL}$ ) in the blood laboratory follow up results. Brain MRI revealed high signal intensities in the right hemisphere of T2 FLAIR, implying seizure-related changes. No meningeal enhancement or basal exudate was observed. An endotracheal tube with mechanical ventilation was placed for respiratory failure, and the patient was admitted to the intensive care unit. Vasopressor (norepinephrine $2 \mathrm{~mL} / \mathrm{h} . \mathrm{kg}$ ) was infused for hemodynamic instability, which originated due to the provisional septic condition. Continuous EEG monitoring and continuous 1-Hz peridodic lateralized epileptiform discharges were detected (Fig. 1B). To prevent hemodynamic instability and RSE, ketamine was administered at a dose of up to $3 \mathrm{mg} / \mathrm{kg} / \mathrm{h}$. Continuous EEG monitoring revealed epileptiform discharges in spite of ketamine infusion. Epileptic discharges terminated immediately on starting low-dose midazolam at $0.08 \mathrm{mg} / \mathrm{kg} / \mathrm{h}$ while on ketamine infusion. During continuous combination infusion, the mean arterial pressure was stable, and norepinephrine was tapered out (Fig. 2). Concomitant AEDs (lacosamide, phenobarbital, perampanel, and clobazam) were added by tapering out the continuous anesthetic drug infusion. Overlapping of these drugs was ensured for approximately 3 days with successful seizure control. The patient's mental status gradually improved, and he was discharged from the hospital after 6 weeks of treatment with antibiotics for pneumonia. At the time of discharge, he was administered two AEDs including lacosamide and phenobarbital.

\section{Discussion}

Our cases showed that early combination therapy of third line anesthetic drugs, having different mechanisms that enhanced GABA activation by blocking the NMDA receptor, is effective in RSE treatment; moreover, this early combination therapy requires only a low dosage of anesthetics, which is suitable for hemodynamic instability. RSE is a neurological emergency and a serious illness with a high risk of mortality and morbidity, especially in elderly patients and those with cardiac comorbidities. ${ }^{8}$ Anesthetics have been introduced to terminate seizure rapidly in RSE and super-RSE; however, hypotension is a critical side effect when treating SE using a higher dosage of benzodiazepines and other $G A B A_{A}$, which often require vasopressor agents. Among anesthetics, NMDA receptor antagonists, such as ketamine, could be efficient in prolonged RSE, while maintaining stable blood pressure by increasing the blood pressure. ${ }^{9}$ Catecholamine reuptake blockade renders ketamine a favorable alternative to anesthetics with negative hemodynamic profiles. ${ }^{9}$ In the pathophysiology of self-sustaining SE, fol- 
lowing repeated seizures, the synaptic membrane of GABA, which is internalizes as clathrin-coated vesicles, inactivates the receptors since they are no longer within reach of the neurotransmitter. In contrast, in NMDA synapses, subunits are mobilized to the synaptic membrane and assembled into additional receptors. As a result of this trafficking, the number of functional NMDA receptors per synapse increases, whereas the number of functional $\mathrm{GABA}_{\mathrm{A}}$ receptors decreases. ${ }^{10}$ Considering the pathomechanism of $S E$ and the side effect of anesthetics, ketamine would be favorable for SE treatment. In our cases, hemodynamic benefits of ketamine combination therapy were obtained with prompt cessation of seizures, with decreased inotropic requirement. Moreover, this combination required a low dosage of midazolam. The well-known potential disadvantages of ketamine are hallucinations and sympathetic adrenergic effects that result in increased intracranial pressure. ${ }^{11}$ However, recent study showed that ketamine has no effect on the intracranial pressure in patients with RSE. ${ }^{12}$ Moreover, the neuroprotective effect of ketamine has been under investigated, and numerous scientific data have been accumulated. ${ }^{13}$ Considering the varied mechanism of ketamine, more prospective clinical trials are warranted to determine the optimal time to early treatment, dosing protocol for ketamine-midazolam combination, and ketamine-midazolam combination use in special conditions, including hemodynamic unstable state.

\section{Conflict of Interest}

The authors declare that they have no conflicts of interest.

\section{References}

1. Legriel $S$, Oddo M, Brophy GM. What's new in refractory status epilepticus? Intensive Care Med 2017;43:543-6.

2. Nelson SE, Varelas PN. Status epilepticus, refractory status epilepticus, and super-refractory status epilepticus. Continuum (Minneap Minn) 2018;24:1683-707.

3. Joshi S, Rajasekaran K, Hawk KM, Chester SJ, Goodkin HP. Status epilepticus: role for etiology in determining response to benzodiazepines. Ann Neurol 2018;83:830-41.

4. Amengual-Gual M, Sánchez Fernández I, Wainwright MS. Novel drugs and early polypharmacotherapy in status epilepticus. Seizure 2019;68: 79-88.

5. Sabharwal V, Ramsay E, Martinez $R$, et al. Propofol-ketamine combination therapy for effective control of super-refractory status epilepticus. Epilepsy Behav 2015;52(Pt A):264-6.

6. Tasker RC, Goodkin HP, Sánchez Fernández I, et al. Refractory status epilepticus in children: intention to treat with continuous infusions of midazolam and pentobarbital. Pediatr Crit Care Med 2016;17:968-75.

7. Fujikawa DG. Starting ketamine for neuroprotection earlier than its current use as an anesthetic/antiepileptic drug late in refractory status epilepticus. Epilepsia 2019;60:373-80.

8. Tatlidil I, Ture HS, Akhan G. Factors affecting mortality of refractory status epilepticus. Acta Neurol Scand 2020;141:123-31.

9. Hurth KP, Jaworski A, Thomas KB, Kirsch WB, Rudoni MA, Wohlfarth $\mathrm{KM}$. The reemergence of ketamine for treatment in critically ill adults. Crit Care Med 2020;48:899-911.

10. Chen JW, Wasterlain CG. Status epilepticus: pathophysiology and management in adults. Lancet Neurol 2006;5:246-56.

11. Höfler J, Trinka E. Intravenous ketamine in status epilepticus. Epilepsia 2018;59 Suppl 2:198-206.

12. Alkhachroum A, Der-Nigoghossian CA, Mathews $E$, et al. Ketamine to treat super-refractory status epilepticus. Neurology 2020;95:e2286-94.

13. Bell JD. In vogue: ketamine for neuroprotection in acute neurologic injury. Anesth Analg 2017;124:1237-43. 